\title{
Frontières
}

\section{Mort et stéréotype : leurs sombres épousailles}

\section{Daniel Castillo Durante}

Volume 19, numéro 2, printemps 2007

\section{Penser sa mort?}

URI : https://id.erudit.org/iderudit/017493ar

DOI : https://doi.org/10.7202/017493ar

Aller au sommaire du numéro

Éditeur(s)

Université du Québec à Montréal

ISSN

1180-3479 (imprimé)

1916-0976 (numérique)

Découvrir la revue

Citer cet article

Castillo Durante, D. (2007). Mort et stéréotype : leurs sombres épousailles. Frontières, 19(2), 23-26. https://doi.org/10.7202/017493ar

\section{Résumé de l'article}

Cet article vise à penser le rapport à la mort à partir d'une réflexion sur ce qui, au sein du langage, paraît, à bien des égards représenter, en abyme pour ainsi dire, la logique qui sous-tend le phénomène de la mort. Dans cette perspective, le stéréotype constitue le mécanisme qui incarne le mieux la tendance vers la pétrification et l'extinction du langage. La présente réflexion fait l'hypothèse que le stéréotype préside à l'élaboration des discours gelés, c'est-à-dire des énoncés dévitalisés, vidés en quelque sorte du nerf créatif sans lequel le langage s'instrumentalise et finit par s'étioler à force de ne déboucher que sur une parole de bois. Là résident les sombres épousailles entre la mort et le stéréotype. 


\section{Résumé}

Cet article vise à penser le rapport à la mort à partir d'une réflexion sur ce qui, au sein du langage, paraît, à bien des égards représenter, en abyme pour ainsi dire, la logique qui sous-tend le phénomène de la mort. Dans cette perspective, le stéréotype constitue le mécanisme qui incarne le mieux la tendance vers la pétrification et l'extinction du langage. La présente réflexion fait I'hypothèse que le stéréotype préside à l'élaboration des discours gelés, c'est-à-dire des énoncés dévitalisés, vidés en quelque sorte du nerf créatif sans lequel le langage s'instrumentalise et finit par s'étioler à force de ne déboucher que sur une parole de bois. Là résident les sombres épousailles entre la mort et le stéréotype.

Mots clés: mort - langue - stéréotype répétition.

\section{Abstract}

This article considers the relationship with death by reflecting on an element of language that in many ways seems to be a representation (en abyme as it were) of the process that underlies the phenomenon of death. Stereotypes are the mechanism that best embodies the tendency of language towards petrifaction and extinction. The hypothesis here is that stereotypes produce a "frozen" sort of speech, lifeless statements that are somehow cut off from the creative vigour without which language becomes instrumental and ultimately withers, engendering nothing but cant. This is the consummation of the "dark nuptials" between death and stereotype.

Keywords: Death - Language Stereotype-Repetition.

\section{MORT ET STÉRÉOTYPE : leurs sombres épousailles}

Daniel Castillo Durante, Ph. D., écrivain et professeur, Département des lettres françaises, Université d'Ottawa.

Comment comprendre le rapport à la mort à partir d'une réflexion sur ce qui, au sein du langage, me paraît, à bien des égards, représenter, en abyme, la logique qui sous-tend le phénomène de la mort? Dans cette perspective, le stéréotype constitue le mécanisme qui incarne le mieux à mes yeux la tendance vers la pétrification et l'extinction du langage. Par hypothèse, le stéréotype préside à l'élaboration de discours gelés, c'est-à-dire des énoncés dévitalisés, vidés en quelque sorte du nerf créatif sans lequel le langage s'instrumentalise et finit par s'étioler à force de ne déboucher que sur une parole de bois. Nous attribuons au stéréotype une fonction régulatrice des discours. Placée entre la topique et la rhétorique - telles que comprises par Aristote -, cette fonction serait capable d'intégrer dans un même mouvement dialectique les réservoirs du déjàdit (clichés, proverbes, maximes, refrains, divers syntagmes figés, métaphores lexicalisées, etc.) et les agencements d'une éco- nomie topique des discours. Le stéréotype développe, dans cette optique, une sorte de force centripète à l'abri de laquelle il se dérobe tout en absorbant l'Autre comme emprunt. L'Autre se reporte ici au clivage du sujet qui se voit de la sorte escamoté. Le stéréotype bouche le vide qui découvre le caractère fondamentalement scindé du sujet parlant qui devient sujet promis à la mort, sujet cliché.

L'origine typographique du mot cliché - en tant que procédé industriel - à l'aube du XIX ${ }^{\mathrm{e}}$ siècle en dit long sur ses rapports pour le moins suspects avec la multiplication des copies conformes d'une société vouée aux techniques du Progrès. Le copiage, ou l'imitation servile, y trouve le moyen d'enraciner un modèle. Face au produit unique - animé par sa propre originalité, métaphore tout court de la vie dans ce qu'elle a de plus spécifique s'érige dorénavant l'exemplaire en série, la caricature du Même, une sorte de clone programmé justement pour remplacer le côté non reproductible de la vie, son aspect le plus authentique. C'est alors que la mise à mort de l'unique nous apparaît comme une condition de possibilité de la 
Modernité. Le cliché, paradigme s'il en est de la Modernité, réfléchissant à l'infini les compositions d'une reproduction industrielle ne pouvait que dévaloriser ou tout au moins banaliser le résultat du procédé. Cette re-production issue de la mécanique même du «Progrès » n'était rien d'autre que l'activité industrielle mise sur le compte du profit. La copie dévoile une perte, qui est celle de l'authentique. L'image maculée se fait ainsi «cliché », c'est-à-dire qu'à sa naissance l'empreinte de la prolifération machinale fait peser sur elle les stigmates du déjà-vu, et les signes de la mise à mort de l'unique. Le machinisme de la copie, point de départ de la consommation massive du cliché, découvre la répétition comme premier moteur d'une altérité en trompel'œil, celle du stéréotype tout autant que celle de la mort.

C'est l'apparition du poncif à l'endroit exact où la copie trahit le Même comme faussaire de sa propre image. Ce ressassement du Même pourra ainsi être stocké, emmagasiné, étalé sur des rayons, devenir marchandise. Il lui faudra néanmoins attendre six décennies avant d'entrer dans la langue (1869) avec le sens figuré et péjoratif d' "idée ou expression trop souvent utilisée ». Le dictionnaire qui constitue précisément le lieu muséal (et d'empaillage tout à la fois) par excellence des clichés décline comme suit leur identité:

Cliché. n. m. (1809; p. p. de clicher).

1) Typogr. Plaque portant en relief

la reproduction d'une page de composition, d'une image, et permettant le tirage de nombreux exemplaires.

Reproduction avec un mastic

formant cliché. V. Polycopie. Cliché en alliage, en plomb. Cliché de cuivre (V. Galvano), de zinc. Cliché en caoutchouc, en plastique. Cliché métallique d'une photographie. V. Héliogravure, photogravure. 2) Phot. (1865). Image négative. V. Négatif. Un cliché net, vigoureux. Copie d'un cliché.

V. Contretype. 3) (1869) Fig. et péj. Idée ou expression trop souvent utilisée. V. Banalité, lieu (commun), poncif, redite. Une conversation pleine de clichés (Le Petit Robert, 2003).

Dans l'arbitraire de sa dictio, le dictionarium cliche (c'est le cas de le dire) l'espace miné à l'intérieur duquel se doit d'œuvrer le mot. N'est-il pas déjà assez contradictoire que ce soit un monument écrit, le point de référence par rapport auquel l'action de dire (dictio) est indexée? En subordonnant de la sorte les variations d'une valeur orale à celle d'une fossilisation

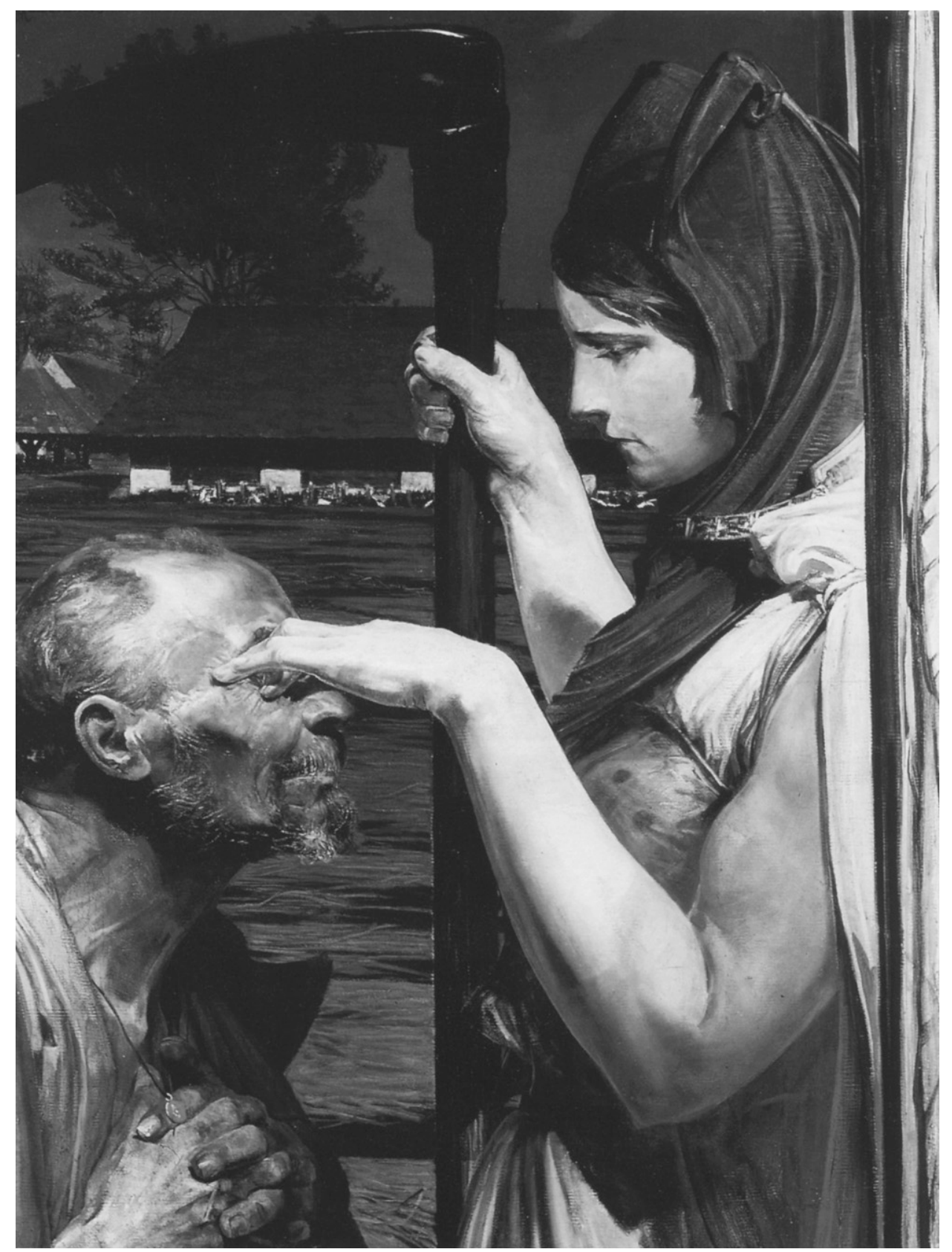

légitimée par l'institution, l'on a affaire à un processus qui n'est pas étranger aux mécanismes liés à la pulsion de mort. Faute donc de pouvoir saisir le mot dans le vif de sa spécificité, le dictionnaire l'épingle. Il fait de lui un otage qui, privé de nerf, doit se contenter d'un statut d'objet empaillé. Cet archivage du mot assure la permanence du système. Aseptisé, dépouillé, parqué dans un ghetto - le ghetto lexicographique -, il est expliqué aux enfants-lecteurs que nous sommes. On nous associe à des renvois qui brouillent son altérité: "Voir: Banalité, lieu (commun), poncif, redite. Une conversation pleine de clichés. » Sur ce broyage, le dictionnaire édifie son entreprise lexicographique. Il y a là un rejet de la signifiance attachée à la lexie qui, séparée de sa gangue sociale, est comme un poisson hors de l'eau: la trace à peine de ce que fut une agonie, une lutte. Le dictionnaire, archiviste et fossilisateur tout à la fois, nie la production du mot en interaction; il escamote son caractère agonique sans lequel le signe se pétrifie.

Il faut comprendre la logique sousjacente à la répétition si l'on veut mettre au jour les mécanismes du stéréotype qui président à l'émergence des unités d'emprunt (p. ex. les clichés). La perspective psychanalytique devient ici indispensable dans la mesure où elle éclaire le caractère fondateur de la répétition. En nous rappelant que «répétition n'est pas reproduction», Lacan (1973) nous évite de voir, dans la répétition, une sorte de fonction purgative du sujet qui s'affranchirait ainsi d'un quelconque besoin. À juste titre, Lacan insiste sur le fait que le concept de répétition chez Freud n'implique «aucun 
retour au besoin», car «le retour du besoin vise à la consommation mise au service de l'appétit». En rejetant donc l'image de la reproduction de la scène primitive (fonctionnant ici comme une sorte de cliché cathartique), on empêche que la répétition ne soit réduite à une pure fonction naturelle, élargissant le problème de la répétition au-delà du cadre de la psychanalyse lacanienne en faisant appel à l'interaction entre la temporalité et la mémoire comme donnée essentielle à la constitution d'un sujet stéréotypal. Bornons-nous pour l'instant à accorder à la répétition, conformément à son acception théâtrale, un statut ludique. Ainsi située, elle devrait m'aider à appréhender, dans un deuxième temps, l'étendue de la scène sur laquelle le stéréotype livre ses jeux de ficelles. L'enfant à la bobine en bois avec une ficelle attachée autour que Freud (1988) s'amusait à observer m'oriente forcément vers le côté ludique d'une représentation faisant $\mathrm{du}$ «partivoilà » (fort-da en allemand) le lieu d'une mise en scène. La trappe de l'Être - son absence - y peut devenir l'obsession du sujet. Pour que la répétition puisse se transformer en "compulsion de répétition », il faudra au père de psychanalyse passer par une étape ludique. On sait que l'enfant en question compensait, grâce précisément à ce jeu (la bobine tenant lieu de signifiant non présent), l'absence de sa mère. Or Freud s'interroge sur le sens de l'acte de l'enfant qui répète comme jeu une expérience douloureuse qui va, bien entendu, à l'encontre du principe de plaisir censé régler les processus psychiques. Il en déduit, non sans quelques hésitations, que «l'enfant ne pourrait répéter dans son jeu une impression désagréable que parce qu'un gain de plaisir d'une autre sorte, mais direct, est lié à cette répétition" (Freud, 1988, p. 55). Il laisse ainsi en suspens le rapport de la répétition au jeu sans se livrer à un développement majeur.

Une étude plus poussée du jeu des enfants ne ferait pas pour autant cesser notre hésitation entre deux conceptions. On voit bien que les enfants répètent dans le jeu tout ce qui leur a fait dans la vie une grande impression, qu'ils abréagissent ainsi la force de l'impression et se rendent pour ainsi dire maîtres de la situation. Mais, d'autre part, il est bien clair que toute leur activité de jeu est influencée par le désir qui domine cette période de leur vie: être grand, pouvoir faire comme les grands. On observe aussi que le caractère déplaisant de l'expérience vécue ne la rend pas toujours inutilisable pour le jeu.

Il n'en demeure pas moins que l'on est en droit d'y voir les bases de l'économie qui régit la répétition chez Freud. Elles relèveraient, me semble-t-il, d'une dialectique entre le principe de plaisir et le principe de réalité. Or cette étude fondamentale de Freud ne s'arrête pas là. Avant même de s'aventurer dans ce que lui-même qualifie de "spéculation» (une spéculation qu'on pourrait à bien des égards qualifier de philosophique), Freud étudie les interactions entre la répétition, le refoulé et la remémoration. En parlant du malade (le névrosé), Freud souligne qu'«il est bien plutôt obligé de répéter le refoulé comme expérience vécue dans le présent au lieu de se le remémorer comme un fragment du passé » (Freud, 1988, p. 57-58). En étudiant ces trois éléments, Freud s'aperçoit que les résistances éprouvées pendant la cure ne doivent nullement être situées au niveau du refoulé mais de son origine. C'est à partir de là qu'il va privilégier le rapport entre ce qu'il appelle le moi et le refoulé. Il s'empresse néanmoins d'ajouter qu' « une grande part du moi est elle-même incons- ment pas inutile de signaler que le mot danois gjentagelse utilisé par le philosophe englobe, outre l'acception de répétition, celle de reprise. L'arrière du ressouvenir et sa projection en avant paraissent ainsi instaurer la logique agonistique qui met en branle l'activité psychique du sujet en tant que projet:

Répétition et ressouvenir sont le même mouvement, mais en sens opposé; car ce dont on se ressouvient, a été; c'est une répétition en arrière; la répétition proprement dite, au contraire, est un ressouvenir en avant. Aussi la répétition, si elle est possible, assure-t-elle le bonheur de l'homme, tandis que le ressouvenir fait son malheur, supposé, bien entendu, qu'il prenne le temps de vivre et ne se mette pas en quête dès l'heure de sa naissance d'un prétexte pour s'évader de la vie, en s'avisant par exemple qu'il a oublié quelque chose (Kierkegaard, 1972, p. 2-3).

LE STÉRÉOTYPE PRÉSIDE À L'ÉLABORATION DE DISCOURS GELÉS, C'EST-À-DIRE DES ÉNONCÉS DÉVITALISÉS, VIDÉS EN QUELQUE SORTE DU NERF CRÉATIF SANS LEQUEL LE LANGAGE S'INSTRUMENTALISE ET FINIT PAR S'ÉTIOLER À FORCE DE NE DÉBOUCHER QUE SUR UNE PAROLE DE BOIS.

ciente, précisément ce que l'on peut nommer le noyau du moi ». Cet aspect difficile à cerner du moi (son atomisation, pour ainsi dire), pose la clef de voûte de l'édifice psychanalytique - la division radicale du sujet - comme borne incontournable pour la problématique du stéréotype. Le mécanisme particulièrement complexe de la répétition (l'on sait que Lacan en fera l'un des quatre concepts fondamentaux de la psychanalyse) doit dès lors être étudié dans son rapport à l'Autre.

La dimension de la temporalité inscrite dans la répétition rappelle inéluctablement la réflexion de Kierkegaard. Le philosophe danois fut l'un des premiers à avoir compris l'importance de la répétition comme pierre angulaire de la pensée moderne. Déjà en 1843, grâce à la lecture de la théodicée de Leibniz, il parvenait à dégager ce qui serait l'un des thèmes majeurs de son ouvre; les conditions de possibilité de ce qu'il appelait la «vie» lui apparaissaient liées au mécanisme de la répétition. Aussi la différence fondamentale que son œuvre autorise entre répétition et ressouvenir permet-elle d'articuler la première à l'intérieur d'une dynamique qui pousse le dernier vers l'avant. Il n'est probable-
Kierkegaard voit dans ce ressouvenir en avant le levier indispensable à un engagement existentiel. Cette formule de la philosophie sartrienne que j'applique ici a le mérite de mettre en relief le caractère éthique revêtu par le mot répétition tel qu'il s'inscrit au tout début de la réflexion de Kierkegaard:

Si Dieu lui-même n'avait pas voulu la répétition, le monde n'aurait jamais été. Ou bien il aurait suivi les plans faciles de l'espérance, ou bien il aurait repassé toutes les choses en son esprit en les gardant dans le ressouvenir. Il ne l'a pas fait; aussi le monde est-il une réalité qui subsiste et dure du fait qu'il est une répétition. La répétition: voilà la réalité et le sérieux de la vie (Kierkegaard, 1972, p. 5).

Or rien ne serait plus faux que d'y souscrire sans tenir compte de l'élasticité dialectique (les mots sont de Kierkegaard lui-même) qui complexifie la notion de répétition chez le philosophe danois. Le concept parvient de la sorte jusqu'à une sorte d'émiettement dans le cadre d'une temporalité parfaite. Cette élasticité de la notion s'explique donc par une approche 
philosophique du temps qui me paraît indispensable pour l'étude du stéréotype. C'est au sommet de sa crête dialectique que la réflexion de Kierkegaard saisit le temps de la répétition comme figure d'une éternité face à laquelle l'homme demeure en suspens.

Comme la mort, le stéréotype ne découvre que des corps gelés alors que la logique interne du phénomène demeure en retrait. En effet, je pars de l'hypothèse que le stéréotype préside à l'élaboration des discours fossilisés, pétrifiés, bref clichés. J'entends par discours clichés l'ensemble d'énoncés dont la cohérence au niveau de leur production de sens obéit à une tutelle stéréotypale. Ce dernier aspect me permet de poser le problème en termes de principe. Apparaît à présent la pertinence d'une réflexion en profondeur. Le stéréotype en tant que principe présuppose un rôle régulateur de l'économie des discours. Bien qu'aucun discours n'échappe à cette règle ${ }^{1}$, on doit demeurer prudent quant au statut qu'il sied d'accorder au terme principe. Parmi les contraintes qui pèsent sur les mécanismes inhérents au marché des échanges d'unités d'emprunt, celle d'une adaptation aux réalités des valeurs en vigueur dans le social me paraît relever d'une logique économique. À l'intérieur de ce cadre, je vois le stéréotype comme principe régulateur. Ceci ne le met nullement à l'abri d'un rapport dialectique avec les unités d'emprunt (clichés, lieux communs, poncifs, métaphores lexicalisées et redites, en particulier) qu'il réactive. Tout se passe comme si, une fois l'agencement topique mis en marche, le stéréotype devait se ménager des voies afin de ne pas être pris dans les représentations qu'il génère. Car le stéréotype comme principe régulateur ne prélève ses gains qu'à assurer une économie de la répétition. Toutefois, rien ne serait plus faux que de voir dans les unités d'emprunt une conformité absolue à cette économie. En faisant appel aux mécanismes de l'emprunt, elles peuvent déstabiliser le système. Il y a de fortes chances pour qu'elles finissent même par entrer en conflit avec le stéréotype. D'où le caractère dialectique ${ }^{2}$ que j'attribue, en dernière analyse, au type de rapport qu'ils entretiennent. Les stratégies d'emprunt qui précèdent le surgissement des unités d'emprunt peuvent s'avérer quelquefois ambiguës ou contradictoires, voire erronées.

La notion d'économie de la répétition ne prétend que rendre compte du stade d'équilibre régressif vers lequel tend le stéréotype. Le stéréotype apparaîtrait ainsi comme l'élément qui assurerait au langage sa transition vers l'extinction tout en mettant en place une économie qui, d'un autre côté, permet la reprise de l'Autre

sous la forme de la copie. Ce paradoxe, voilà mon hypothèse, rejoint celui de la mort. La mort comme principe régulateur d'une économie de la répétition serait cette altération qui fait basculer le sujet dans l'anonymat d'un lieu commun, sorte de dépotoir de la reconnaissance. La mort comme reconnaissance ultime serait ainsi

COMME LA MORT,

\section{LE STÉRÉOTYPE NE DÉCOUVRE}

QUE DES CORPS GELÉS

ALORS QUE LA LOGIQUE INTERNE

$\frac{\text { DU PHÉNOMÈNE }}{\text { DEMEURE EN RETRAIT. }}$

celle d'une stéréotypie qui fait peser sur le sujet le joug de sa mortalité comme trait spécifique de l'humain. Pure sommation muette et masquée, tout comme le stéréotype, la mort évite le nom car, dans sa force aveugle de transfert, elle ne reconnaît personne. En révoquant la souveraineté de notre conscience individuelle, elle nous assujettit au lieu commun mais libère en même temps ces restes à partir desquels il nous faudra dorénavant interpréter le monde. Interpréter le monde à partir de la dépouille du père est au demeurant le geste fondateur de la loi. C'est probablement ce rapport de la mort à la loi qui fonde, d'un point de vue phénoménologique, le stéréotype (stereos, pater), c'est-à-dire le stereos, le solide, le dur, du typus («modèle», «symbole»), ou le tupos («empreinte», «marque»). En ce sens, le stéréotype, tout comme la mort, est un geste qui se répète; dans le silence glacé des saisons, il révèle le nom comme lieu de représentation truquée, demeure du factice où le sujet affirme une identité qui le rattache à un ordre symbolique que la mort gomme. Les cimetières avec leurs épitaphes gravées dans la pierre sont là pour que le visiteur, rare et parfois pressé, fasse l'appel de mémoire onomastique que lui dicte une conscience souvent coupable. Cet abattoir de noms ayant mordu la poussière illustre à certains égards l'impasse d'une société qui a du mal à penser la mort autrement que par des mécanismes, c'est le cas de le dire, de pétrification. La fosse commune s'érige ici comme le dernier outrage à la mémoire du défunt. Rien ne désespère davantage l'homme occidental que l'anonymat de la mort car elle nargue son besoin pérenne de clicher tout ce qui lui échappe. En ce sens, la mort se présente comme margi- nale, en bordure de la société du spectacle (Debord, 1987, 1988). Elle opère dans un registre aléatoire qui ignore le principe d'assurance qu'exige la société occidentale pour continuer à « rouler». L'anonyme de la mort installe le précaire, le stochastique et le marginal comme alternatives proprement finales face à une programmation qui, paradoxalement, finit par faire de la vie un réseau d'accès à des reconnaissances de plus en plus fragmentaires. Les mots de passe devenant proliférants, le seul password qui se dérobe à notre emprise technologique est précisément celui de la mort dont l'anonymat nous nargue et nous assujettit à une attente anxieuse et souvent crispée. Hostile à une véritable mise à l'épreuve de l'Anonyme autre, la société occidentale en est à se souhaiter une mort rapide et indolore. La dimension épistémologique, la sommation de son altération, y sont donc passées sous silence. Or si pensée de la mort il y a, elle ne peut se faire que dans un espace de détachement qui exige une révocation en doute des représentations généralement en noir et blanc de ce phénomène extrême.

Ces sombres épousailles entre le stéréotype et la mort sont pensées ici à partir d'une perspective heuristique qui cherche à dévoiler des passerelles entre le langage, la répétition et le phénomène extrême par lequel la conscience du sujet est suspendue à une révocation qui relève proprement de l'ordre de l'impensable. Car, en effet, la mort - grâce au stéréotype - confie au cliché, à l'image toute faite, gelée en somme, le soin de représenter cette chute de l'humain dans les dépotoirs du «on meurt», lieu commun, indéclinable.

\section{Bibliographie}

DEDORD, G. (1988). Commentaires sur la société du spectacle, suivi de la Préface à la quatrième édition italienne de La société du spectacle, Paris, Gallimard.

DEBORD, G. (1987). La société du spectacle, Paris, Éditions Lebovici.

FREUD, S. (1988). Au-delà du principe du plaisir, Paris, Payot.

KIERKEGAARD, S. (1972). La répétition, Paris, Éditions de l'Orante.

LACAN, J. (1973). Le Séminaire, Livre XI, Les quatre concepts fondamentaux de la psychanalise, Paris, Seuil.

\section{Notes}

1. Tout discours étant, en effet, passé au pressoir des grilles topiques mises en place par le stéréotype.

2. Il s'agit d'un rapport qu'on peut qualifier d'interagonistique dans la mesure où il $\mathrm{y} a$ lutte et engagement d'un processus de reconnaissance de part et d'autre. 